\title{
Computer Modeling
}

National Cancer Institute

\section{Source}

National Cancer Institute. Computer Modeling. NCI Thesaurus. Code C16461.

A technique which attempts to provide an abstract model of a particular system. It

utilizes a mathematical model, which attempts to predict the behavior of the system from a set of parameters and initial conditions. 